\title{
Client-Server and Peer-to-Peer Ad-hoc Network for a Flexible Learning Environment
}

\author{
$\underline{\text { doi:10.3991/ijim.v5i1.1444 }}$ \\ Ferial Khaddage ${ }^{1}$, Christoph Lattemann² \\ ${ }^{1}$ Deakin University, Melbourne, Australia \\ 2 Jacobs University, Bremen, Germany
}

\begin{abstract}
Peer-to-Peer (P2P) networking in a mobile learning environment has become a popular topic of research. One of the new emerging research ideas is on the ability to combine P2P network with server-based network to form a strong efficient portable and compatible network infrastructure. This paper describes a unique mobile network architecture, which reflects the on-campus students' need for a mobile learning environment. This can be achieved by combining two different networks, client-server and peer-to-peer ad-hoc to form a sold and secure network. This is accomplished by employing one peer within the adhoc network to act as an agent-peer to facilitate communication and information sharing between the two networks. It can be implemented without any major changes to the current network technologies, and can combine any wireless protocols such as GPRS, Wi-Fi, Bluetooth, and 3G.
\end{abstract}

Index Terms-Peer-to-Peer, client-server, network, mobile learning.

\section{INTRODUCTION}

Mobile Learning is on the rise with the upcoming of new quasi cost-free wireless technologies (flat-rates); The Importance of mobile learning is increasing not only for off-campus learning environment but also for on-campus as well. Mobile learning has shown to be effective in sharing and transferring learning content among students and is often effective when used to build mobile student groups to form an on-campus mobile learning environment [1].

Mobile Peer-to-Peer network is already offering crucial and unique opportunities for innovations especially at universities, and can advance the delivery mechanism of learning content.This is not only true for off-campus students, but also for on-campus students who require wireless access to course content. Therefore this network can provides easy, reliable and free access to the learning content while on-campus.

With the emerging of high bandwidth wireless channels such as Third Generation Network (3G) widely installed the wireless Local Area Network (Hotspots). Also with the advancements of the new mobile devices and the provision of new business models by the telecommunication providers, which is based on flat-rate, the broad usage of mobile phones for retrieving and accessing learning content at universities is becoming more feasible and is on the rise.

Wireless technologies and mobile networks are constantly changing and they are being developed at a rapid speed, hence, new possibilities are emerging that can be used along the $3 \mathrm{G}$ telecommunication network.

The network infrastructure and architecture are normally achieved by designing high-availability networks which take a unique approach to the subject by covering the ideas underlying networks, the architecture of the network elements, and the implementation of these elements are considered crucial aspects. This paper describes mobile network architecture and applied technology that is capable of providing wireless access to learning content within the university campus. Also wireless protocols suitable for mobile devices such as Wi-Fi, Bluetooth and $3 \mathrm{G}$ are being explained. Concluding remarks, advantages and limitations will be presented at the end of this paper.

\section{Wireless Protocoll FOR MoBile PhONES}

The rapid development of mobile devices especially mobile phones for communication purposes, have triggered its application in educational institutions. Also the software trend towards ad hoc and peer-to-peer networks and the ability to target mobile phone devices for networked applications for the delivery of learning content, offers a great opportunity for higher education institutions and in particular for universities.

In the following sections we discuss mobile network architecture which comprises a State-of-the-Art communication technologies and wireless networks for mobile learning purposes. Some of them are already being developed at universities and in particular Deakin University. The main focus lies on the local wireless networks (WLAN), as this is the network which mostly used by student to access learning material and for the delivery of e-learning content. Wireless Local Area Network (WLAN) is normally free of charge especially when accessed by students while on-campus, and it is safe and secure, because the university offers a secured network, and has implemented all security measures to prevent unauthorised login attempts.

In the following sections we discuss the wireless network protocols:

\section{A. Third Generation Network (3G)}

Number Smart phones use a mobile network technology to deliver and receive different types of data. Information technologist, system and network developers classify this technology into generations. With the advancement and the rapid development of the mobile phones an advanced protocols were created and they are the $2 \mathrm{G}$ and the $3 G$ networks. 3G system must allow simultaneous use of speech and data services, and provide peak data rates of at 
least $200 \mathrm{Kbit} / \mathrm{s}$, this is according to the IMT-2000 specification. 3G networks consist of cells; each cell contains a base station using a number of radio frequencies or channels. The mobile phone is always communicating with the network via those channels. These channels consist of two types, one to handle verbal data, audio and voice communication, and the other to manage signalling and messaging, therefore users can receive a massage when they are unable to make a call or when the call drops [2] [3].

GPRS (General Packet Radio Service) is a wireless communication service which can be classified as a 2G Technology. This protocol is used mostly for smart phones communication. It is a packet-switched, "always on" connection that remains active as long as the phone is within range of the service. It allows smart phones to do things like run applications remotely over a network, interface with the Internet, participate in unsynchronized communication sessions, and it does act as a mobile modem for a computer and transmit and receive e-mails. GPRS can send and receive data at a rate of 114 kilobytes per second [4]. It provides moderate speed data transfer, by using unused Time Division Multiple Access (TDMA) channels in the GSM network. GPRS offers up to 171.2 Kbps, depending on the network availability, channel coding scheme and terminal capability [4]. However, in practice, the bit rate is $40-80 \mathrm{Kbit} / \mathrm{s}$. But this bandwidth will only provide poor quality to user for real-time streaming and communication.

GPRS is penetrating the mobile network at a rapid speed. Almost every mobile in the market support GPRS and GPRS services like Multimedia Messaging Service (MMS) and Wireless Application Protocol (WAP) are widely used. To use it, it is necessary to re-implement the Wireless Service Subsystem. Point-to-Point (PTP) service will be used to discover an active device and peer-to-peer transfer. Also, the higher the data rate, the lower the error correction capability. Generally, the connection speed drops logarithmically with distance from the base station. This is not an issue in heavily populated areas with high cell density like a university environment, but may become an issue if access attempt are made remotely [5].

\section{B. The Wi-Fi Technology}

A wireless network uses radio waves that communicate in two ways: a centralized network with access point, called infrastructure network and, a decentralized network without access point, called ad-hoc network. Despite that Wi-Fi power consumption is considered high, still this technology is the most widely used especially at universities. This is because Wi-Fi allows connectivity in peer-topeer mode, which enables devices to connect directly with each other. Hence, in the context of education at universities, learning content can be wired with higher bandwidth.

The area around a Wi-Fi access point is considered as a hotspot. Universities as well as several companies and institutions have already created their own hotspot, for example Deakin University, McDonald restaurants, Starbucks, to enable users to access the Internet via a partly free of charge Wi-Fi network.

Most of the mobile phones today are embedded with Wi-Fi; therefore student can use their mobile phone to access the Wi-Fi network [4]. This type of connectivity is useful in mobile learning environment.
The Wi-Fi radio transmission happens at frequencies of $2.4 \mathrm{GHz}$ or $5 \mathrm{GHz}$, which is considered very high and allows the signal to carry more data [6]. Wi-Fi uses a license-exempt portion of the radio spectrum so that everyone can set up his own Wi-Fi network without applying for a license or paying for broadcasting rights. Also, Wi-Fi can provide the high bandwidth for good quality audio streaming. There are currently three main specifications 802.11, 802.11a, 802.11b and 802.11g in the family of IEEE 802.113.2 [7].

All three specifications use the Ethernet protocol and Carrier Sense Multiple Access with Collision Avoidance (CSMA/CA) for path sharing.

\section{Bluetooth}

Bluetooth is a specification for a mobile wireless Personal Area Networks (PANs), also known under the IEEE 802.15.1 standard [8]. Bluetooth is a rapidly growing technology [9], focusing on building Personal Area Networks (PANs) and connecting mobile devices cheaply and wirelessly. Bluetooth is available in multiple classes with varying power requirements, and varying range. These specifications make the ideal connection within an ad hoc peer-to-peer networking environment. Fig.1 is an illustration of the location of Bluetooth within the wireless network. It is also a brief illustration of the three main categories in wireless protocols, which are $2 \mathrm{G}$ and $3 \mathrm{G}$ wireless networks, WLAN and LAN and the location of the Bluetooth technology lies.

Bluetooth can offer many functionalities, it enables different mobile devices to connect and exchange data and information. Bluetooth is designed for low power consumption within a short range of $1-100$ meters with a data transmission rate of up to $24 \mathrm{Mbit} / \mathrm{s}$. It is based on low-cost transceiver microchip which does exist in almost each mobile device [14].

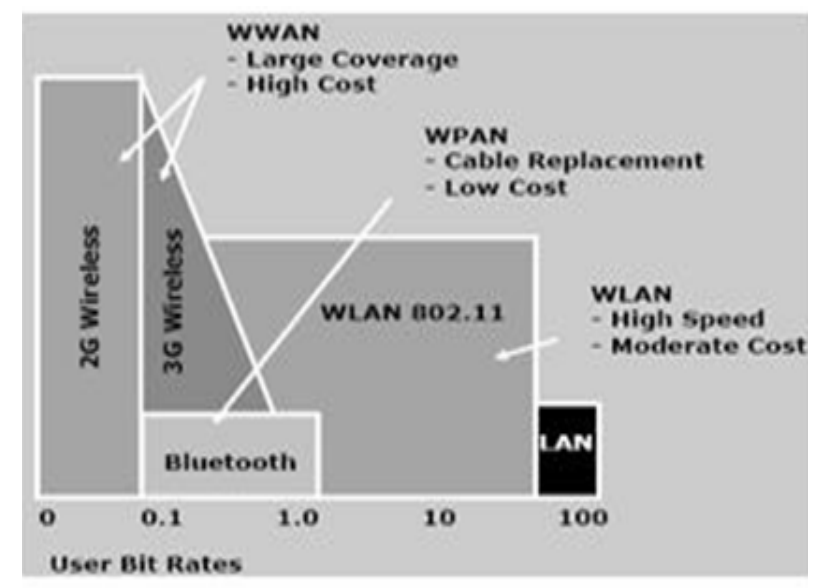

Figure 1. Wireless Protocols Feature [9]

Therefore Bluetooth allows mobile phones to communicate with one another as long as they are within range. Since Bluetooth is a radio communication system, mobile phones do not have to be in line of sight of each other, unlike the requirement for infrared information transfers.

Therefore Bluetooth, if used, will enable the system to be portable, flexible, and cost effective. Cost is an important aspect to consider, because communication via a mobile phone can still be expensive, and charges for downloads by the network carriers are often quite expen- 
sive. However, on the other hand communication and sharing resources via Bluetooth doesn't cost the student anything, as it is normally free of charge [9].

Also there are three different connection types available for Bluetooth see table1 below

TABLE I

TYPES OF BLUETOOTH CONNECTIONS

\begin{tabular}{|c|c|}
\hline Connection identifier & Expanded name \\
\hline \hline Btgoep & General object exchange profile \\
\hline btl2cap & Logical link control and adaption layer protocol \\
\hline Btspp & Serial port profile \\
\hline
\end{tabular}

First, btgoep transmits binary data, so it is not appropriate for string-based communication. And btl2cap requires more work from developers, setting and working around maximum message sizes. However, btspp hides these complexities from the developer, so this is the logical connection choice for a project aimed at simplifying our development process [9].

Therefore this makes Bluetooth transport layer connectivity to client applications without overburdening the client with code and asynchronous messaging considerations. This will let us achieve a straight forward factoring of function and leave code simple to develop hence low to maintain [9].

\section{THE PROPOSED NETWORK ARCHITECTURE}

As previously discussed there are different types of mobile networks, each one of them comes with different characteristics, properties, advantages and disadvantages for their users.

Therefore in order to identify which one of them is suitable and most likely to be adapted and can be used in different contexts, we had to briefly review their features before making our selection.

For the implementation of a network for mobile learning purposes at universities, we have to consider certain requirements from students such as price sensitivity, service and technology availability, ease of use and usefulness. A mobile learning environment should allow peer to peer communication as well as up-load and down-load to and from a central server. In this respect Bluetooth has the advantage of being widely used in most mobile phones [14], and it is available free of charge to all users, thus making transferring multimedia files like learning images and material cheap, easy [15] and convenient [10].

Furthermore, mobile phones with Bluetooth are normally problems free, establishing connection depends also on the operating system, supported specification and programming interface [15]. Bluetooth is also considered one of the possible wireless protocols to allow sharing and transferring of information in an ad-hoc learning environment. GPRS and Wi-Fi could be used as well. But GPRS requires students to subscribe for the service and pay for the download content, and Wi-Fi on the other hand requires higher power consumption, which also comes at a high cost for student. All this makes Bluetooth connectivity the most convenient and most suitable for mobile learning purposes in a university setting.

Via the wireless network, mobile phones can detect and locate the nearby devices with other students on campus, hence making sharing and uploading images and learning materials from the server to student's mobile phone fast and efficient, thus making learning more interactive, portable, collaborative and flexible. This will be explained further in the next section.

\section{A. Overview of the Network Infrastructure}

Our proposed architecture is implemented as a peer-topeer mobile network, but also creates an "ad-hoc" mobile social network. Also these kinds of networks can be integrated together with the already existing client-server systems. They are called Ad-hoc Messaging Network (AMNET) [12][13].This network is different from the normal peer-to-peer (p2p) connection as it does offer a connection to the server. This connection is achieved via allocating one peer to act as an agent, and the communication between the P2P ad-hoc network and the client-server network is normally handled by the peer agent that acts as a facilitator of the communication network and offer other peers a service to interact with the connected server. The agent peer behaves like a normal peer, but it recognizes its specific role during the runtime. Because of this self managing there is no need to configure the agent peer, as it acts like a proxy for the main server, thus making the server an extra service in the P2P network. The illustration of the combined P2P and client-server network is displayed in fig. 2. and fig. 3

This would enable content exchanged between two different network types [11]

Therefore we do not have to do many changes to the already existing network at the university. And the transformation takes place easily and effectively in minimum time. In fact, the student will not notice any difference between the deliveries.
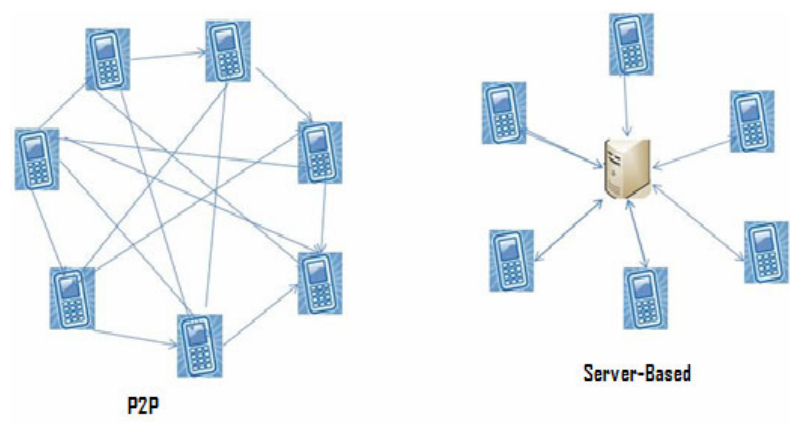

Figure 2. Peer-to-Peer Vs Server-based Network

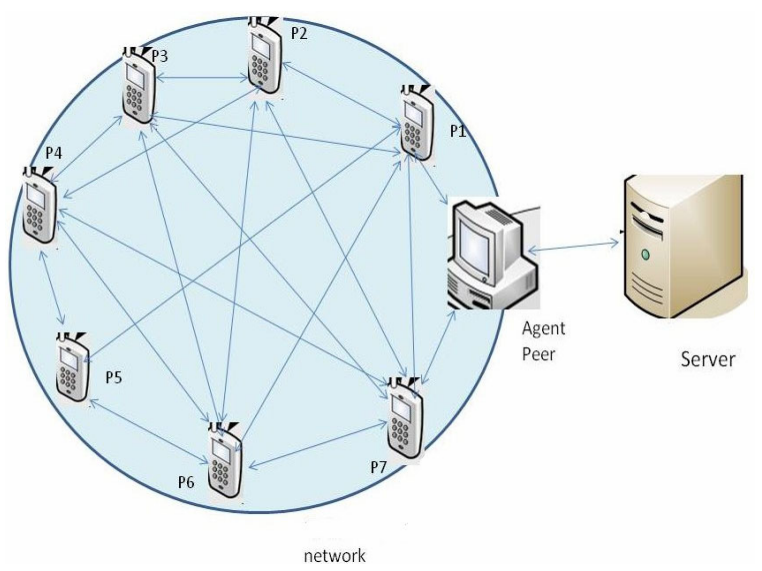

Figure 3. Agent peer to facilitate P2P and Client Server Network 
For our system we pursue an approach which follows few good techniques within AMNet. Firstly by keeping routing as simple as possible, and secondly by considering the content as the point of interest and deny the ambition to bring all internet features to mobile networks, this is also achieved by considering both networks, client-server and ad-hoc peer-to-peer. Our approach is to separate the two different networks by a peer client, and allocate it as an agent peer to facilitate data transmission between the two networks, hence providing a best-fit solution mechanism for certain applications' requirements.

Therefore the proposed network follows the AMNet (Ad-hoc Messaging Network) approach [12], but the implementation of the peer agent to integrate to our existing client-server network is unique, effective and efficient, especially when all method of communications between the two networks must pass through the agent peer first, so any security threat will be handled by the agent peer before it actually reaches the vulnerable wireless network.

By deploying this method we are forming a strong secure network infrastructure that is capable of combing both networks effectively and efficiently. As a matter of fact this technique would fit seamlessly within the already existing network client-server structure at the university and can wrap the two networks together in an efficient manner, thus making communication and transmission of material among clients and server fast, reliable effective and secure.

\section{B. Advantages of the Proposed Network}

In general this type of network infrastructure can provide benefit to the university as well as to the students themselves, especially because the connection is achieved via an Ad-hoc Bluetooth network connectivity where any mobile phone embedded with Bluetooth can communicate and share resources free of charge. Fig. 3 shows that this type of network can help to support speedy access and efficient retrieval of the learning content, between peer-topeer and server and Peers via the agent peer.

Therefore mobile network of devices using Bluetooth technology protocols is to allow one host device to interconnect with many active client devices (because a threebit MAC address is used). Up to 255 further client devices can be deactivated, or parked, which the host device can bring into active status at any time. Also material can be transferred between the host and one client, but the host switches rapidly from server to agent peer and from client to client. Now to set up a connection, a client would perform an inquiry to find any available device to connect, and any host can be configured to respond to such inquiries. The host requires its owner to accept but the connection itself can be held until it goes out of range. Full illustration of this network and its protocol are beyond the scope of this paper and will be available in future publications.

To sum up our proposed architecture provides the following advantages for mobile learning in a university setting:

- By connecting both networks, (Ad-hoc peer-to-peer and client-server), the limitation of each particular network will be overridden by the alternative network.
- Each client on the network is free to share its own resources with other client and at the same time able to connect to the server via the peer agent.

- Since p2p network is not centralized, thus making administration difficult, therefore all administrations are handled on the client-server side.

- Cost is reduced as the client-server network already exists, and the integration of p2p network can be established at a low cost.

- It offers more security, since security related issues are handled by the client-server, and data must pass the peer client before it reaches each client on its own.

- As a method of proper mobile phone integration within the current learning environment.

- This architecture and concept guarantees portability, flexibility and availability of the learning content, when this content is saved on the mobile phone, and could be then transferred, shared and copied.

\section{CONCLUSION}

Mobile phones and their networks are shaping our future; they are fusion technology that is capable of providing what once considered science fiction.

The proposed network architecture is not a replacement for any other delivery mechanisms, but rather an extension or an addition to the learning environment. The new mobile learning environment is to strengthen the other learning methods at university, by providing a mobile access to learning content and at the same time gives students the freedom of choice on selecting an alternative mobile method to access learning content.

This paper illustrated a valuable communication and network for a vivid ad hoc network that can be integrated with a server-based network. It also depicted an approach towards a technical solution that realizes all requirements for combining client-server and peer-to-peer ad-hoc network.

However, the limitation of this approach is that a lot of data have to be stored on the mobile device, and this sometimes can form a barrier due to limited storage space, limited capacity and less functionality in earlier versions of some mobile devices. But with the advancement of mobile devices and their rapid innovative development, for example the recent release of the iPad, iPhone 4, the blackberry mobile phone and many others, are already overcoming this limitation and will continue to do so in the in the near future.

\section{REFERENCES}

[1] Khaddage F., Lanham E. and Zhou W. (2009)"A Mobile Learning Model for Universities, -Re-blending the Current Learning Environment"International Journal of Interactive Mobile Technologies. ISSN: 1865-7923

[2] Rich Mogull, "Peering Inside a Mobile Phone Network" Networking and Communication website. Available online from http://db.tidbits.com/article/9796 [Last accessed on 12 May 2010]

[3] Voda Phone Official Website. Available online from http://www.vodafone.com/hub_page.html [Last accessed on 11 April 2010]

[4] Cell phones with Wi-Fi coming before end of this year, Available online from http://msmobiles.com/news.php/3097.html [Last accessed 24 October 2009] 
[5] Smith K. and Cap O. "Impact on Social Change: Benefits and Barriers to School Culture and the Integration of M-Technology". International Journal of Interactive Mobile Technologies (iJIM) Volume 2, Issue 1, January 2008.

[6] Bettstetter C. ,Vogel H. J. ,Eberspacher J. ,(1999) VGSM Phase 2+ General Packet Radio Service GPRS: Architecture, Protocols, and Air Interface, Technische Universität München (TUM)

[7] EDGE, World record data speed on T-Mobile Hungary's GSM EDGE network supplied by Ericsson,( 2004), Available online from http://www.ericsson.com/ericsson/press/releases/20040702951738.shtml [Last accessed 23 April 2009]

[8] IEEE 802.15, Standard Association. Wireless Personal Area Networks, available online from http://standards.ieee.org/getieee802/ 802.15.html [Last accessed 23 April 2010]

[9] Liscano R. (2006) Introduction to Bluetooth Networking Carleton University. Available online from http://www.site.uottawa.ca/ $\sim$ rliscano/presentations/IntroToBT.pdf [Last accessed 24 April 2010]

[10] Bygrave C. (2009) IBM Java Technology Center (JTC) (United Kingdom). Developing IBM's Java 2 Platform, Standard Edition (J2SE) implementations.

[11] Gradecki, J. D., editor (2002). Mastering JXTA: Building Java Peer-to-Peer Applications. Wiley Publishings, Indianapolis, USA.

[12] Khaddage F. , Lattemann C. (2009) "Towards an Ad-hoc Mobile Social Learning Network Using Mobile Phones" The $12^{\text {th }}$ International Conference on Interactive Computer Aided Learning, ICL 2009 proceedings, September 2009, Villach, Austria ISBN: 978-389958-481-3
[13] Fuchß C. Stieglitz S. and Hillmann O. (2006) "Ad-hoc Messaging Network in a Mobile Environment," presented at International Conference of Internet Technology and Secured Transactions, London, 2006.

[14] Kaasinen, E (2005) "User acceptance of mobile services - value, ease of use, trust and ease of adoption" VTT PUBLICATIONS Technical Research Centre of Finland 566

[15] Tourrilhes, J. (2003) On-Demand Bluetooth: Experience integrating Bluetooth in Connection Diversity, Research Paper Hewlett Packard Laboratories

\section{AUTHORS}

Dr Ferial, Khaddage is $\mathrm{PhD}$. Lecturer of Computing and Information Technology at Deakin University, Faculty of Science and Technology, School of Information Technology, 221 Burwood Highway Burwood Victoria 3125 Australia (faye.khaddage@deakin.edu.au)

Prof. Dr Christoph, Lattemann is Professor of Business Administration and Information Management at Jacobs University, School of Humanities and Social Science business Administration Campus Ring 128759 Bremen Germany, (c.lattemann@jacobs-university.de).

Submitted September $9^{\text {th }}$, 2010. Published as resubmitted by the authors December $11^{\text {th }}, 2010$. 